\title{
Modeling behavior of decision makers with the 2 aid of algebra of qubit creation-annihilation \\ operators
}

\author{
Polina Khrennikova,
}

University of Leicester, University Road, Leicester LE1 7RH. pk198@le.ac.uk

October 12, 2016

\begin{abstract}
We present a general model of the process of decision making based on the representation of the basic behavioral variables with the aid of an algebra of qubit creation-annihilation operators, adopted from the quantum information theory. In contrast to the genuine quantum physical systems, which are divided into either bosons or fermions and modeled with the aid of operators, satisfying canonical commutation or anti-commutation relations, decision makers preferences for possible actions are constructed with the aid of operators satisfying the socalled qubit commutation relations. Systems described by operators, satisfying such commutation relations, combine the features of bosons and fermions. Thus, one of the basic consequences of the presented model is that decision makers mimic the combined bosonic-fermionic behavior. By using the algebra of qubit creation-annihilation operators, we proceed with the construction of the concrete operators, describing the process of decision making. In particular, the generators of the quantum Markov dynamics, which is used for modeling human decision making process, are expressed as polynomials of the qubit creation-annihilation operators. The devised coefficients have a natural cognitive and social meaning.
\end{abstract}

Keywords: Decision making, decoherence, quantum-like model, quantum master equation, qubit creation-nnihilation operators, (anti-)commutation relations. 


\footnotetext{
${ }^{1}$ At the same time, see Plotnitsky (2014), Boyer-Kassem et al. (2016a, b) for a critical analysis of the ability of quantum formalism to cover all problems arising in mathematical modeling of human reasoning and decision making.

${ }^{2}$ To be consistent with the above notations for the position and momentum operators, we should proceed with the symbols $\hat{a}^{\star}, \hat{a}$, where in the quantization formalism the hats symbolize the operator nature of quantities. However, to simplify notation in long expressions for operators which will be constructed as polynomials of the creation-annihilation
} 
operators, we shall skip the hats.

${ }^{3}$ In theoretical quantum computing researchers operate with unitary gates and in real physical applications they have to move either to bosonic or fermionic algebras. 


\footnotetext{
${ }^{4}$ For example, for decision making in finance such an environment contains the information on the real state of economics, world-wide political news, as well as psychological factors, such as expectations of investors related to future price formation on the finance market. In the context of decision making by voters, an election environment contains information related to the economic and finance conditions, political news, but also a variety of psychological biases conveyed by the mass-media during the election campaign (Khrennikova et al., 2014, 2016, Khrennikova, 2014a, 2015, 2016).
} 
Consider a number of agents $\mathcal{A}^{i}, i=1, \ldots, n$. They plan some actions with respect to each other; possible actions of $\mathcal{A}^{i}$ with respect to the agents $\mathcal{A}^{j}, i \neq$ $j$, are given by the variable

$$
X_{i}=x_{1} \ldots x_{i-1} x_{i+1} \ldots x_{n} ;
$$

\footnotetext{
${ }^{5}$ Of course, this is a statement about the general state of affairs. One cannot exclude a possibility that in some decision making contexts agents' behavior might be in accord with the purely bosonic or fermionic statistics. Finding such empirical examples, e.g., in cognitive psychology, economics, game theory would be of a vast interest. We remark that fermionic creation-annihilation operators were applied by Bagarello $(2012,2015)$ and Bagarello and Haven (2016) to model creation of alliances between political parties and the dynamics of buying and selling of financial assets. We also point to exploring of the Fock space formalism for modeling of cognitive phenomena by Sozzo (2014). A more detailed description of the mathematics and social meaning of fermionic and bosonic operators can be found in the appendix.

${ }^{6}$ However, such two dimensional anyons are not real physical particles. They are the so called quasiparticles.
} 
in the simplest case $x_{j}=0,1$, for example, non-cooperate/cooperate, notbuy/buy securities or commodities. In general, we obtain

$$
x_{j} \in\left\{\alpha_{1}, \ldots, \alpha_{q}\right\},
$$

where the possible actions $\alpha$ can depend on an agent, i.e., for the agent $\mathcal{A}^{i}, q=q^{i}$ and $\alpha_{k}=\alpha_{k}^{i}$

This formalization is able to cover a variety of problems in psychology, decision making in social settings, behavioral economics and finance, corporate finance and political science. Below are some decision making examples, including some global decision making contexts. ${ }^{7}$

- The agents are states and the possible actions $x_{j}$ represent the degree of cooperation between them; including decision making in global political contexts, in the form of cooperation/non-cooperation on some political issues.

- The agents are political parties within the same country and the variables $x_{j}$ represent the degree of cooperation between them; for example, in the model with $x_{j}=0,1$, the value $x_{j}=1$ for the political party $\mathcal{A}^{i}$ corresponds to its intention to create a coalition or establish cooperation with a party $\mathcal{A}^{j}$.

- The agents' are trading assets on a finance market, where each of them sells just one type of an asset (stocks of one company) and the variables $x_{j}=0,1$, correspond to decisions on buying, respective not-buying an asset offered by $\mathcal{A}^{j}$. The model can be modified to correspond to the real environment of a finance market, by considering $x_{j}^{i}=\alpha_{1}^{i}, \ldots, \alpha_{q_{i}}^{i}$, where each $\alpha_{j}$ is by itself a portfolio of assets, which $\mathcal{A}^{i}$ can buy from $\mathcal{A}^{j}$. (The counterparts can also make sell/hold decisions).

- The agents are members of a social network (virtual or real) and $x_{j}^{i}$ represent the degree of connectivity of $\mathcal{A}^{i}$ with $\mathcal{A}^{j}$.

- Two companies negotiate entering a merger (i.e. to become one joint company). In the simplest model there are two parties $\mathcal{A}^{1}$ (management

\footnotetext{
${ }^{7}$ We remark that at this stage we do not consider some concrete game theoretic problems, where only cooperation or competition is the best strategy. In the analysed example on political cooperation/competition we do not assume there are some constraints to cooperation, see a detailed synthesis of coalition-entry impact factors in Khrennikova (2016). The decision on cooperation is driven by internal characteristics (value of cooperation shaped by the ideology, power aspirations and other factors) and external environmental impact (feedback from the electorate).
} 
and shareholders of company one) and $\mathcal{A}^{2}$ (management and shareholders of company two) and the problem is related to negotiations between the involved companies. In this setting $x_{j}=0,1$, variables correspond to yes/no in respect to the company's decision on the merger entry.

- A couple $\mathcal{A}^{1}$ (Alice) and $\mathcal{A}^{2}$ (Bob) plans to rent a flat or a house and they select accommodations offered by a few estate agents $\mathcal{A}^{i}, i=$ $3, \ldots, n$. They can select a few flats or houses and sign one for them in their vectors $X_{k}=x_{3} \ldots x_{n}, x_{i}=0,1, k=1,2$, and the agents also have their own vectors with dichotomous coordinates corresponding to all the individuals searching for accommodation and asking for the flats, which have already been selected by Alice and Bob. The decision making problem can be quite complex for both sides, the couple and the estate agents (e.g., the latter can be very careful and to check the financial background of applicants for accommodation). More generally, the coordinates encoding the states of Alice and Bob and the real estate agents do not need to be dichotomous.

The model is non-trivial even for a single agent $\mathcal{A}$ who makes decisions about her possible actions in an informational environment (private, political, social, finance). Here the latter plays the role of the second agent, but we are not interested in the dynamics of its concrete state.

- A trader $\mathcal{A}$ of the financial market should make the decision about buying some financial asset: in the state vector of $\mathcal{A}$, see (1), the coordinates $x_{i}=0,1$, where the index $i$ labels some financial assets.

- A voter $\mathcal{A}$ decides for which party (or a particular candidate) she will vote; the same model is applicable to different sorts of referendums, e.g., $x=0,1$, "to leave EU/to stay in the EU", "Scotland leaves UK/notleaves".

The common feature in all the above selections of actions is that agents act in contexts characterized by uncertainty. As was emphasized in the introduction, the mathematical formalism of quantum theory is able to capture agents' resolution from uncertainty very well. Of course, the classical probability also handles very well a range of choice problems, at the same time decision making paradoxes can emerge. Quantum probability allows for a possibility to capture decision making revealed in such paradoxes e.g., the paradoxes of Ellsberg (1961) and Machina (2009) (Haven and Khrennikov, 2009, Aerts et al., 2012). ${ }^{8}$ We remark that, roughly speaking, the main

\footnotetext{
${ }^{8}$ Of course, a number researchers delivered successful contributions in resolving these
} 
distinguishing feature of quantum probability is that it provides the unique possibility to handle superpositions of the states of agents. In the above examples, we use the notion of "state" in the classical sense. In the next section we will consider quantum states constructed as superpostions of the coordinate vectors given by (1), (2).

\section{Quantum-like representation of states of the agents}

To simplify considerations, we proceed with the case of two possible actions $x=0,1$ for all involved agents. This case will be handled with the algebra of qubit creation-annihilation operators, in section 6 . To describe a general case of non-dichotomous actions, a new operator algebras would need to be introduced. This would be a topic for our further studies.

We consider the space of mental states of decision makers which was introduced in by Khrennikova (2016) in special context of decision making at the political arena. Now we extend this formalism to the general decision making context considered in section 2 . The space of possible actions of the agent $\mathcal{A}_{i}$ towards another (fixed) agent $\mathcal{A}_{j}$ can be mathematically represented (in the quantum-like manner) as one qubit space (two dimensional complex Hilbert space) with the basis $(|0\rangle,|1\rangle)$ encoding agent's preferences: "not/act". It is denoted by the symbol $H_{i j}$. In the quantum-like model uncertainty in $\mathcal{A}_{i}$ 's preferences is represented by superposition of non-action and action. Such superpositions are naturally expressed by (normalized) linear combinations of the states $|0\rangle$ (non-action) and $|1\rangle$ (action):

$$
|\psi\rangle=c_{0}|0\rangle+c_{1}|1\rangle
$$

paradoxes, by using the mathematical tools of classical probability theory, cf. Tversky and Kahneman, (1974, 1981, 1983), Tversky and Shafir (1992), Kahneman and Tversky (2000) for a critical analysis of the classical probabilistic framework of decision making. However, often, a model modifying the expected utility theory and resolving some paradox, e.g., the Ellsberg paradox, becomes an object of new "paradoxical attacks". For example, the original models explaining the Ellsberg paradox were not able to explain the Machina paradox. Now the classical probabilistic approach to decision making is involved in the long-term and endless struggle against appearance of new paradoxes. In the review (Erev and Ert, 2016) one can find 39 paradoxes and, as pointed by the authors of this review, the dream of classical probabilistic theory of decision making is to create a model which would not suffer of any of these known paradoxes. However, one cannot exclude that such a "grand-unification model" would be attacked by creators of a new paradox ("40th paradox") cf. Birnbaum (2008) or Machina (2009). The quantum-like approach pretends to resolve the probabilistic paradoxes of decision making theory in one model. However, for a moment these are just the great expectation, see, however, Asano et al. (2016). 
where $c_{0}$ and $c_{1}$ are complex numbers, $\left|c_{0}\right|^{2}+\left|c_{1}\right|^{2}=1$.

For the fixed agent $\mathcal{A}_{i}$, the complete state space $H_{i}$ is represented (in complete accordance with quantum information theory) as the tensor product the state spaces $H_{i j}$ corresponding to $\mathcal{A}_{i}$ 's preferences for (non-)action towards agents $\mathcal{A}^{j}, i \neq j$. Thus $H_{i}=\otimes_{i \neq j} H_{i j}$. The dimension of this space is equal to $d=2^{n-1}$. This space contains superposition of all possible actions of $\mathcal{A}_{i}$ towards other agents.

The complete decision context involves the preferences for (non-)action of all agents (towards each other). The complete state space is mathematically represented as the tensor product $H=\otimes_{j} H_{j}$. In the qubit representation its vectors have the form:

$$
|\Psi\rangle=\sum_{\mathcal{X}} C_{\mathcal{X}}|\mathcal{X}\rangle,
$$

where $\mathcal{X}=X_{1} \ldots X_{n}$ and, see (1), $X_{j}=x_{1} \ldots x_{j-1} x_{j+1} \ldots x_{n}, x_{j}=0,1$, and $\sum_{\mathcal{X}}\left|C_{\mathcal{X}}\right|^{2}=1$. The dimension of this space is equal to $D_{n}=2^{n(n-1)}$.

In the space $H$ we have both basic quantum effects, superposition and entanglement. In particular, as the result of entanglement the agents "loss their individual control over decisions about (non-)action towards other agents." The action of each agent $\mathcal{A}_{i}$ are irreducibly coupled with possible actions of other agents.

Remark 1. (On "mental superposition") We remark that, in quantum physics, superposition also bears a purely operational meaning. In contrast, to classical physics the notion of superposition does not simply relate to physical waves propagating in physical space-time. The effect of superposition is conveyed via the interference experiments, such as seminal the two slit experiment. As was shown by Feynman (1965), in the purely probabilistic terms such experiments demonstrate a violation of the basic laws of classical probability theory. Thus the results presented in (Busemeyer et al., 2006, 2012; Pothos et al., 2009, Accardi et al., 2008, 2009, Asano et al., 2011ab, 2012, Basieva et al., 2011, Aerts et al. , 2012, Bagarello, 2012, 2015; Khrennikova et al., 2014, 2016, Khrennikova, 2014a, b, 2015, 2016) demonstrate violation these probabilistic rules for some effects observed in cognitive psychology. Some well-known effects are order, conjunction and disjunction effects that call for the usage of an alternative approach to decision making. In fact, the usage of the formalism of states superposition (operationally encoded in the complex linear space representation) adopted from quantum formalism in cognition, psychology, decision making offers a viable alternative mathematical decision making framework. However, recall that in quantum-like models (as well as in quantum physics) the notion of superposition is an operational mathematical tool, i.e., we do not associate it with th existence 
of some "mental waves". Formally, a measurement (decision making, action, answer) reduces superposition to one of the basis states corresponding to this measurement. This reduction is often called a state collapse. Again we regard the notion of collapse operationally (although in physics there are a few theories of "physical collapse"), cf. with White et al. (2013, 2014, 2015), especially White et al. (2014).

\section{Mental entanglement}

In the mathematical language entanglement is defined as the impossibility to represent a state belonging to the tensor product $H$ of a few Hilbert state spaces $H_{j}, j=1,2 \ldots, m$, in the factorized form, i.e., as the tensor product of the components belonging to the tensor factors $H_{j}$ of $H$.

\subsection{Interpretation}

In this paper (similarly to superposition), entanglement is treated as an operational tool which is used in the Hilbert space representation of correlations between observables.

The main message of quantum physics (theory and experiment) is that here correlations can be stronger than in classical physics (violation of Bell's inequality and its generalizations). There can be mentioned two main sources of the "quantum amplification" of correlations:

- nonlocal action at a distance;

- the impossibility of objectivization quantum observables: one cannot assign the values to incompatible quantum observables before experiment.

The latter is very natural for cognition: there is no reason to assume that an individual has somewhere in her brain the answers to all possible questions which were "prepared in advance". For example, the otder effect says us that such in advance preparation is impossible. The same can be said about the disjunction effect (Tversky and Shafir,1992) expressing a violation of the Savage Sure Thing principle (Savage, 1954). We recall that the quantum-like approach to decision making was very successfully used in the mathematical modeling of these effects (Busemeyer et al., 2006, 2012, Conte et al., 2007, 2009, Pothos and Busemeyer, 2009, Wang and Busemeyer, 2013). In fact, the model of dynamical decision making which was elaborated by Busemeyer et al. (2006, 2012) and Pothos and Busemeyer (2009) explores fundamentally 
quantum entanglement, although these authors did not underline explicitly this important feature of their model. However, they work in the four dimensional Hilbert space (for the game with two players) and starting with a factorizable (i.e., not entangled) pure state they then produce entanglement by the specially selected unitary rotation in the four dimensional Hilbert space. ${ }^{9}$ More generally the framing effect which was very well studied in cognitive psychology (Tversky and Kahneman, 1981, Kahneman and Tversky (2000)) also can be treated a sign of non-objectivity of mental observables. And it can be used as the simplest explanation of entanglement of cognitive entities.

Surprisingly we cannot neglect even the nonlocal dimension in interpretation of entanglement. Of course, we do not mean the mystical action at a distance which would provide the possibility of instantaneous update of mental states of people located far from each other. (Such an action would be useful to explain parapsychological effects.) We consider just the possibility of signaling between decision makers or in the brain of a single decision maker. In physics the main problem is that if such a signaling were existing it has to be too rapid or even instantaneous. There were performed experiments demonstrated that if this action were propagated with a finite velocity, it should to be many times larger than the velocity of light. In cognitive studies we know well that information processing in the brain has a finite velocity and this cognitive time scale provides the possibility of signaling inside the brain - between its different parts. Similarly decision makers, e.g., the traders at the financial market, use optical fiber connections and the velocity of inter-agent signaling approaches the velocity of light. Hence, such purely classical nonlocality can contribute to mental entanglement and, in particular, in strengthening of quantum correlations.

Thus both nonobjectivity of mental observables and signaling between agents and inside the brain can contribute to generation of special states for groups of agents or even decisions of a single agent which are mathematically described as entangled. However, even in physics the notion of entanglement is one of the most complicated from the interpretational viewpoint. Its complete clarification would need additional tremendous efforts. For a moment, the best strategy is just pragmatically use the mathematical formalism of quantum theory. In such an approach entanglement cannot be "explained", but only confirmed by experiment ${ }^{10}$ We point out that the Bell type tests

\footnotetext{
${ }^{9}$ This entanglement generating rotation is constructed phenomenologically by using the elements of the payoff matrix.

${ }^{10}$ We pinpoint that in QM entanglement between quantum systems does not necessarily need to imply non- locality, if one adopt the view of local realism, cf. works by Loubenets (2012), Loubenets (2015).
} 
for functioning of cognition are not easy to perform (but the same can be said about physics: the final Bell test without loopholes was performed only in 2015). However, some preliminary results have already been obtained, see (Conte et al., 2008, Asano et al., 2014). We also point to studies of Dzhafarov and Kujala $(2012,2014)$ on application of the quantum formalism and especially entanglement to psychophysics and its coupling with studies about selective influences which have been very well studied in psychophysical literature.

\subsection{Monogamy of entanglement}

Monogamy of entanglement (for $n \geq 3$ ) is one of its distinguishing features. In the case of a pure state (i.e., given by a normalized vector) it is formulated very simply. Consider the case of three agents (e.g., political parties acting at the political arena of some country) $\mathcal{A}_{1}, \mathcal{A}_{2}, \mathcal{A}_{3}$. We call entanglement between their preference states genuine tripartite entanglement, if the their preference state cannot be bi-separated, i.e., it cannot be represented, e.g., in the form:

$$
|\Psi\rangle=\left|\Psi_{12}\right\rangle \otimes\left|\Psi_{3}\right\rangle,
$$

where $\left|\Psi_{12}\right\rangle \in H_{1} \otimes H_{2}$ is an entangled state and $\left|\Psi_{3}\right\rangle \in H_{3}$. We remark that the state (5) need not be factorizable into three states. Thus if the state $\Psi_{12}$ is not factorizable, then the state $\Psi$ is entangled (in spite of partial separability).

The mathematical formalism of QM implies the following "monogamy" feature of entanglement. Suppose that the preferences of agents $\mathcal{A}_{1}, \mathcal{A}_{2}, \mathcal{A}_{3}$ are entangled. If, e.g., $\mathcal{A}_{1}$ and $\mathcal{A}_{2}$ share an entangled pure state $\Psi_{12} \in$ $H_{1} \otimes H_{2}$, then they cannot have any entanglement with $\mathcal{A}_{3}$, regardless of how weakly entangled their state is. Thus if the state of the tri-agent preferences $|\Psi\rangle$ is entangled (and pure) and at the same time the state of one of the bi-agent preferences is also entangled (and pure), then $|\Psi\rangle$ is automatically biseparable.

We have to recognize that entanglement monogamy (for pure states) does not match completely the rules of decision making "games" between a few agents. In general, decision makers are not swans who can have so to say only pairwise entanglement. For example, suppose agents are political parties acting at state's political arena and establishing cooperation of different degree, including creation of alliances, see Bagarello (2015 a,b), Bagarello and Haven (2016) and Khrennikova (2016).

However, this is not a constraint to using the notion of entanglement in quantum-like modeling of decision making. This is merely one of the 
evidences that modeling with the aid of solely pure states is restrictive. One has to proceed with in general mixed states, see also section 5 for another motivation having the dynamical nature.

For in general mixed states, the monogamy of agents' preferences for actions can be formulated as follows: if the entanglement between the two of the three agents (e.g., the political parties) increases, then the entanglement between either of those two and the third (other) agent must decrease. The latter features matches well with the rules of the decision making "games". Two agents (e.g., political parties) cannot increase they inter-connection without decreasing their interconnections with the third agent (political party). However, the latter is definitely not the feature of all possible games between agents. Thus the impact of the monogamy feature of the quantum entanglement to applicability of this formalism in cognition, psychology, and decision making has to be analyzed more carefully, cf. (Plotnitsky, 2014, Boyer-Kassem et al., 2016a, b). It seems that the role of the monogamy issue of quantum entanglement has not been risen in previous papers about the quantum-like modeling of decision making. It might happen that entanglement monogamy would constraint applicability of the quantum formalism in cognition, psychology, sociology, economics, or finance.

\section{Quantum-like schemes for modeling of de- cision making}

Following the ideology of the quantum-like modeling of the dynamical process of decision making (Busemeyer et al., 2006, 2012; Pothos and Busemeyer, 2009; Asano et al., 2011, 2012; Bagarello, 2012, 2015, Bagarello and Haven, 2016) we describe the process of decision making with the aid of the quantum state dynamics.

To model the process of decision making Busemeyer et al. (2006, 2012), Pothos and Busemeyer (2009), Zorn and Smith (2011) and a few other authors used the standard quantum scheme: continuous Schrödinger evolution interrupted by measurement - in our case a discontinuous act of selection of the concrete alternative for decision making. Bagarello $(2012,2015)$ modeled the dynamics of averages by using the quantum field version of the unitary Schrödinger dynamics, see also Bagarello and Haven (2016). Asano et al. (2011ab, 2012), Basieva et al. (2011) proposed to apply the decoherencemeasurement scheme based on quantum master equation. In political science this scheme was applied by Khrennikova et al. (2014, 2016) and Khrennikova (2014a, b, 2016, 2016). In this paper we also apply this scheme. Our aim is 


\footnotetext{
${ }^{11}$ In decision-making modeling, environment is treated broadly compromising of the set of mental, economic, financial, social, geo-political and ecological variables.
} 
and interpret it as the factor determining the time scale of the dynamics of the state of decision maker in the process of selection of possible actions.

For natural generators of dynamics, the solution of the GKSL-equation (6), the time dependent density operator $\rho(t)$, approaches for $t \rightarrow \infty$ the steady state $\rho_{\text {out }}$. This steady state is considered as the output of the process of agents' decision making. The diagonal elements of the density operator $\rho_{\text {out }}$ in the basis corresponding to possible actions, see section 3, encode the probabilities of possible actions. In the simplest case the density operator acts in the two dimensional qubit space. The operator $\rho_{\text {out }}$ encodes the probabilities of actions labeled by $\alpha=0,1: p_{\alpha}=\left\langle\alpha\left|\rho_{\text {out }}\right| \alpha\right\rangle$. As in classical decision making, the problem of interpretation of probabilities arises (Plotnitsky, 2009, Haven and Khrennikov, 2016) ). They can be interpreted either as objective (frequency) probabilities as in von Neumann and Morgenstein (1953) or as subjective probabilities, cf. Savage (1954). We proceed with a subjective interpretation. Now, as in the classical decision making, to select the concrete action $\alpha=0,1$, the decision maker calculates the odds: $O(1)=\frac{p_{1}}{p_{0}}$. If $O(1)>1$, she selects the action $\alpha=1$, in the opposite case, she selects the action $\alpha=0$. (If $O(1)=1$, she will continue analysis of the problem or just select the action purely randomly.)

We point to the main distinguishing feature of the decision making model based on the GKSL-equation. In contrast to the classical von NeumannMorgenstern expected utility approach and its numerous generalizations (we use the umbrella EUT), our agents do not directly appeal to utility of choices. The agent's utility function is not part of the model. In the above model, an agent does not seek to maximize expected utility in the strict EUT meaning. An agent makes her decision by taking into account information gained from interaction with the environment and her internal cognitive features. The procedure of decision making is not as straightforward as in the expected utility approach. The decision state $\rho_{\text {out }}$ is approached in the process of stabilization of fluctuating preferences for a set of actions and the dynamics of such fluctuations can be very complex ${ }^{12}$.

\footnotetext{
${ }^{12}$ We would like to illuminate that the internal characteristics" encoded in the decisionoperators can contain a set of variables that corresponds to the value/utility interpretation of human actions as understood in EUT. At the same time, there is a set of additional variables characterising biases, beliefs and memory, cf. a concrete illustration with a projected structure of the Hamiltonian operator by Pothos and Busemeyer (2009).
} 


\section{What are the features of agents' decision making? Bosonic? Fermionic? Qubits?}

We recall once again that all quantum physical physical systems are either bosons or fermions and mathematically are described by canonical commutation and anti-commutation relations respectively. At the same time quantum information theory is basically done in $n$-qubit space. It is well know that qubit is neither boson nor fermion (Frydryszak, 2011). In some sense it combines both fermionic and bosonic features. Thus in quantum information theory the qubit representation is a mathematical model which does not represent the real physical situation. Therefore to have the real physical model one has to transfer the theory written in qubit terms either to the bosonic or fermionic representation and it is possible to do (Bravyi and Kitaev, 2002).

In the quantum-like model of decision making qubit by itself is a basic entity of a quantum-like model. We need not to transfer the $n$-qubit model neither into bosonic nor fermionic one. Therefore we cannot proceed with canonical (anti-) commutation relations and explore advantages of the standard formalism of creation and annihilation operators (for bosons or fermions). Instead of the standard formalism, we have to use the qubit canonical commutation relations which combine nilpotence of fermionic creation and annihilation operators with commutativity of the corresponding bosonic operators.

Consider single qubit space with the basis $(|0\rangle,|1\rangle)$. We define here the standard fermionic operators of creation $a^{\star}$ and annihilation $a$ as following:

$$
\begin{gathered}
a^{\star}|0\rangle=|1\rangle, a^{\star}|1\rangle=0 \\
a|0\rangle=0, a|1\rangle=|0\rangle,
\end{gathered}
$$

or in the matrix representation

$$
a^{\star}=\left(\begin{array}{cc}
0 & 0 \\
1 & 0
\end{array}\right), a=\left(\begin{array}{cc}
0 & 1 \\
0 & 0
\end{array}\right) .
$$

Hence, $a^{\star}$ is really the adjoint operator to $a$. These operators satisfy the canonical commutation relations:

$$
\left\{a, a^{\star}\right\}=I,\{a, a\}=0,\left\{a^{\star}, a^{\star}\right\}=0,
$$

where $I=\left(\begin{array}{ll}1 & 0 \\ 0 & 1\end{array}\right)$, is the unit operator and the anti-commutator of two operators $A$ and $B$ is defined as $\{A, B\}=A B+B A$. The commutation relations (10) can be easily checked by using the matrix representation (9). 
Here the last two commutation relations in (10) are in fact trivial, since $\left(a^{\star}\right)^{2}=a^{2}=0$. The number operator can be represented in the standard way $N=a^{\star} a$ and the free Hamiltonian as $\mathcal{H}_{0}=\omega a^{\star} a$. We remark that in quantum information theory (Frydryszak, 2011) these anti-commutation relations are written in the following form [?]:

$$
\left[a, a^{\star}\right]=I-2 N,\left(a^{\star}\right)^{2}=a^{2}=0 .
$$

Now we want to proceed to the case of a few degrees of freedom, to the $k$-qubit space. Let $W=W_{1} \otimes \ldots \otimes W_{k}$, where $W_{i}$ is one qubit space. In each $W_{i}$ we introduce the operators of creation and annihilation $a_{i}^{\star}, a_{i},(7)$, (8), but then we extend them onto space $W$ in the standard tensor product space manner

$$
\mathbf{a}_{i}^{\star}=I \otimes \ldots I \otimes a_{i}^{\star} \otimes I \ldots \otimes I, \mathbf{a}_{i}=I \otimes \ldots I \otimes a_{i} \otimes I \ldots \otimes I,
$$

i.e.,

$$
\begin{aligned}
& \mathbf{a}_{i}^{\star}\left|x_{1}\right\rangle \otimes \ldots\left|x_{i}\right\rangle \otimes \ldots \otimes\left|x_{k}\right\rangle=\left|x_{1}\right\rangle \otimes \ldots a_{i}^{\star}\left|x_{i}\right\rangle \otimes \ldots \otimes\left|x_{k}\right\rangle, \\
& \mathbf{a}_{i}\left|x_{1}\right\rangle \otimes \ldots\left|x_{i}\right\rangle \otimes \ldots \otimes\left|x_{k}\right\rangle=\left|x_{1}\right\rangle \otimes \ldots a_{i}\left|x_{i}\right\rangle \otimes \ldots \otimes\left|x_{k}\right\rangle .
\end{aligned}
$$

For the fixed $i$, such operators satisfy the canonical commutation relations (10) for the one dimensional fermionic system, but for different $i, j$ they commute:

$$
\left[\mathbf{a}_{i}, \mathbf{a}_{j}^{\star}\right]=\left[\mathbf{a}_{i}, \mathbf{a}_{j}\right]=\left[\mathbf{a}_{i}^{\star}, \mathbf{a}_{j}^{\star}\right]=0,
$$

where $[A, B]=A B-B A$ is the usual commutator. Now we list the $k$-qubit cannonical commutation relation as they are typically written in quantum information theory:

$$
\begin{gathered}
{\left[\mathbf{a}_{i}, \mathbf{a}_{j}^{\star}\right]=\delta_{i j}\left(1-2 N_{j}\right)} \\
{\left[\mathbf{a}_{i}, \mathbf{a}_{j}\right]=0,\left[\mathbf{a}_{i}^{\star}, \mathbf{a}_{j}^{\star}\right]=0,} \\
\left(a^{\star}\right)^{2}=0, a^{2}=0 .
\end{gathered}
$$

Now we turn to our model of decision making. In the total preference state space $H$ of the agents $\mathcal{A}^{i}, i=1,2, \ldots, n$, we introduce the operators $\mathbf{a}_{j i}^{\star}, \mathbf{a}_{j i}, i \neq$ $j, i, j=1, \ldots, n$. For the fixed $j$, the operator $\mathbf{a}_{j i}^{\star}$ creates the preference for action of $\mathcal{A}^{j}$ towards $\mathcal{A}^{i}$ and the operator $\mathbf{a}_{j i}$ destroys it. ${ }^{13}$

\footnotetext{
${ }^{13}$ We underline that the operators create and annihilate preferences and not the actions. We describe the process of decision making and during this process an agent reflects on "to act, or not to act". These reflections are encoded with the aid of the qubit creation and annihilation operators. At the end of the process of reflections an agent approaches the decision which is represented in the probabilistic form and gives (subjective) probabilities for the actions.
} 
We emphasize that these operators are "local", i.e., they nontrivially act only on the corresponding qubit representing the relation of $\mathcal{A}^{j}$ to $\mathcal{A}^{j}$. This feature of the qubit creation and annihilation operators reflects the basic feature of the decision making process, the agent $\mathcal{A}^{j}$ can act to each qubit of its preference state independently from other agents. ${ }^{14}$

\section{Model generators of quantum Markovian dynamics by using qubit creation and an- nihilation operators}

Now we want to present some model operators generating the GKSL-dynamics by using the qubit creation-annihilation operators. As was emphasized in introduction, we cannot start modeling of cognition from a mental analog of the phase-space representation used in classical physics. If we were able to proceed in this way, it would be possible to apply the Schrödinger quantization procedure and replace the canonical variables by noncommutative operators (and by taking into account that decision makers are neither bosons nor fermions, see section 6.) The absence of the mental equivalent of the classical physical phase-space representation is a consequence of the impossibility (may be temporary) to identify "mental canonical variables", the analogs of position and velocity (momentum) of a physical system. In any event, we cannot proceed by using Schrödinger quantization. And the quantization procedure based on the creation and annihilation operators is the most attractive alternative which can be explored. In quantum physics, bosonic and fermionic operators are in use. As was remarked, in quantum information theory one can proceed with qubit creation and annihilation operators. However, up to my knowledge, this formalism is not so widely explored, see, however, again (Frydryszak, 2011).

First, we consider the Hamiltonian part of the dynamics. The dynamics in the absence of interactions between agents and between different preferences

\footnotetext{
${ }^{14}$ In quantum computing this feature corresponds to the possibility of approaching each qubit of the multi-qubit state. One may say that in our model agents use quantum-like algorithmic procedures for decision making. Of course, the state transformation given by the GKSL-equation is not a genuine quantum gate, because the latter has to be represented by a unitary operator and it corresponds to Schrödinger's dynamics. However, in some modern schemes of quantum state control non-unitary gates accommodating the influence of the bath are started to be used.
} 
of a single agent is generated by "free Hamitonian":

$$
\mathcal{H}_{0}=\sum_{j} \mathcal{H}_{0 j}, \mathcal{H}_{0 j}=\sum_{i} \omega_{j i} \mathbf{a}_{j i}^{\star} \mathbf{a}_{j i}
$$

where $\omega_{j i} \geq 0$ are parameters ("frequencies") determining the time scales dynamics of the preference of the agent $\mathcal{A}^{j}$ for (non-)action towards the agent $\mathcal{A}^{i}$ and $\mathcal{H}_{0 j}$ is the Hamiltonian of the agent $\mathcal{A}^{j}$. The latter would describe its preference state dynamics if this agent were evaluating her preferences for (non-)action towards other agents without taking into account "external signals" about preferences of other agents (and this is unrealistic situation).

The interaction Hamiltonian is modeled in the following way (as, e.g., in quantum optics):

$$
\mathcal{H}_{I}=\sum_{j_{1}, j_{2}} \sum_{i_{1}, i_{2}} k_{j_{1} j_{2} i_{1} i_{2}}\left[\mathbf{a}_{j_{1} i_{1}}^{\star} \mathbf{a}_{j_{2} i_{2}}+\mathbf{a}_{j_{2} i_{2}}^{\star} \mathbf{a}_{j_{1} i_{1}}\right],
$$

where $k_{j_{1} j_{2} i_{1} i_{2}}$ are real coefficients describing the magnitude of pairwise interactions. This Hamiltonian is quadratic with respect to the qubit operators of creation and annihilation. Interactions of higher order, e.g., of fourth degree, can also be modeled, but the corresponding equations are too complicated even for numerical modeling.

Now the adjustment of the preferences of the agent $\mathcal{A}^{j}$ as the result of the influence of her mental environment $\mathcal{R}_{j}$ we describe by the operator ${ }^{15}$ :

$$
L_{j} \rho=\sum_{i \neq j}\left[\alpha_{i j}^{+}\left(\mathbf{a}_{j i}^{*} \rho \mathbf{a}_{j i}-\frac{1}{2}\left\{\mathbf{a}_{j i} \mathbf{a}_{j i}^{*}, \rho\right\}\right)+\alpha_{i j}^{-}\left(\mathbf{a}_{j i} \rho \mathbf{a}_{j i}^{*}-\frac{1}{2}\left\{\mathbf{a}_{j i}^{*} \mathbf{a}_{j i}, \rho\right\}\right)\right],
$$

where $\alpha_{i j}^{+}$is a coefficient giving "the rate of signals" in favor of action towards the agent $\mathcal{A}^{i}$ coming to the agent $\mathcal{A}^{j}$ from her mental environment $\mathcal{R}_{j}$ and $\alpha_{i j}^{-}$gives the "rate of signals" against action. It seems to be difficult to determine these rates experimentally, since even the notion of a "signal" has to be specified. For a moment, we consider these coefficients as just quantitative expressions of the environment's pressure to the agent $\mathcal{A}^{j}$ to perform (or not) an action towards the agent $\mathcal{A}^{i}$.

\footnotetext{
${ }^{15}$ We again use the analogy with quantum physics modeling interaction of a multi-level atom with the electromagnetic field. The only difference that we use qubit operators of creation and annihilation $\mathbf{a}_{j i}^{*}$ and $\mathbf{a}_{j i}$.
} 


\section{Actions of political parties towards and against cooperation}

For example, in the previous scheme the role of agents can be played by political parties $\mathcal{P}^{j}, j=1,2, \ldots, n$, see (Khrennikova, 2016) for details. Thus here political parties plays the role of decision makers. Each party considers the problem of cooperation with other parties. ${ }^{16}$ The action is "to cooperate" and each party reflects on preferences on (non-)cooperation. Here the environments $\mathcal{R}_{j}$ are parties' electorates. And the coefficients $\alpha_{i j}^{+}, \alpha_{i j}^{-}$represent electorate's will that the political party $\mathcal{P}^{j}$ would establish the cooperation with the political party $\mathcal{P}_{i}$.

In the operational representation under consideration, the presence of the unstable electorate $\mathcal{R}$ is expressed in adjustment of the rates in the operator (19): $\alpha_{i j}^{ \pm} \rightarrow \alpha_{i j}^{ \pm}+\gamma_{i j}^{ \pm}$. Although from a purely mathematical viewpoint such an adjustment makes no difference, some interesting effects of the presence of the common unstable electorate $\mathcal{R}$ can be modeled. For example, suppose $\mathcal{R}$ strongly wants the cooperation between, e.g., all parties on the political arena. This will is expressed in increase of all $\alpha_{i j}^{+}$by the same additive term $\gamma^{+}$of sufficiently high magnitude. This will modify the preference state dynamics essentially.

Suppose that there are only two political parties, $\mathcal{P}_{1}$ and $\mathcal{P}_{2}$. Each $H_{j}$ is just the qubit space of the dimension two. The preferences to noncooperation and cooperation are represented by the bases $(|0\rangle,|1\rangle)$ in $H_{j}$. The joint states of preferences are represented by superpositions of the vectors from the basis

$$
e_{1}=|00\rangle, e_{2}=|10\rangle, e_{3}=|01\rangle, e_{4}=|11\rangle \text {. }
$$

In this basis the creation and annihilation operators for preferences of $\mathcal{P}_{1}$ and $\mathcal{P}_{2}$ are represented by the matrices or in the matrix representation

$$
\mathbf{a}_{1}^{\star}=\left(\begin{array}{cccc}
0 & 0 & 0 & 0 \\
1 & 0 & 0 & 0 \\
0 & 0 & 0 & 0 \\
0 & 0 & 1 & 0
\end{array}\right), \mathbf{a}_{1}=\left(\begin{array}{cccc}
0 & 1 & 0 & 0 \\
0 & 0 & 0 & 0 \\
0 & 0 & 0 & 1 \\
0 & 0 & 0 & 0
\end{array}\right) .
$$

\footnotetext{
${ }^{16}$ In the quantum-like framework the problem of creation of alliances between political parties was originally considered by Bagarello (2015b) whose model was based on exploration of the mathematical apparatus of quantum field theory, see also (Bagarello and Haven, 2016).
} 


$$
\mathbf{a}_{2}^{\star}=\left(\begin{array}{cccc}
0 & 0 & 0 & 0 \\
0 & 0 & 0 & 0 \\
1 & 0 & 0 & 0 \\
0 & 1 & 0 & 0
\end{array}\right), \mathbf{a}_{2}=\left(\begin{array}{cccc}
0 & 0 & 1 & 0 \\
0 & 0 & 0 & 1 \\
0 & 0 & 0 & 0 \\
0 & 0 & 0 & 0
\end{array}\right) .
$$

The Markovian quantum master equation, the GKSL-equation, has the form

$$
\begin{gathered}
\frac{d \rho}{d t}=-\frac{i}{\gamma}\left[\omega_{1} \mathbf{a}_{1}^{\star} \mathbf{a}_{1}+\omega_{2} \mathbf{a}_{2}^{\star} \mathbf{a}_{2}+k_{12}\left(\mathbf{a}_{2}^{\star} \mathbf{a}_{1}+\mathbf{a}_{1}^{\star} \mathbf{a}_{2}\right), \rho\right] \\
+\left(\alpha_{1}^{+}+\gamma^{+}\right)\left(\mathbf{a}_{1}^{*} \rho \mathbf{a}_{1}-\frac{1}{2}\left\{\mathbf{a}_{1} \mathbf{a}_{1}^{*}, \rho\right\}\right)+\left(\alpha_{1}^{-}+\gamma^{-}\right)\left(\mathbf{a}_{1} \rho \mathbf{a}_{1}^{*}-\frac{1}{2}\left\{\mathbf{a}_{1}^{*} \mathbf{a}_{1}, \rho\right\}\right) \\
\left(\alpha_{2}^{+}+\gamma^{+}\right)\left(\mathbf{a}_{2}^{*} \rho \mathbf{a}_{2}-\frac{1}{2}\left\{\mathbf{a}_{2} \mathbf{a}_{2}^{*}, \rho\right\}\right)+\left(\alpha_{2}^{-}+\gamma^{-}\right)\left(\mathbf{a}_{2} \rho \mathbf{a}_{2}^{*}-\frac{1}{2}\left\{\mathbf{a}_{2}^{*} \mathbf{a}_{2}, \rho\right\}\right) .
\end{gathered}
$$

This is a system of linear equations, its dynamics can be modeled numerically. Behavior of solutions depends essentially on the magnitudes of the coefficients and selection of the initial conditions. We plan to analyze such dependences in a future paper.

\section{Concluding remarks}

We apply the decoherence approach to the quantum measurement to model the process of decision making in the very general setup: multi-agent context, where each agent can assign preferences for possible actions towards some other agents. This generality leads to a complex structure of the multi-agent state space.

This complex Hilbert space representation encodes uncertainty of conditions for selection of possible actions. This uncertainty is encoded in superposition of states corresponding to concrete actions. Such an superposition uncertainty can be interpreted as being more deep and unresolved, than the belief uncertainty modeled in the formalism of classical probability theory by assigning probabilities to the possible actions, see (Busemeyer et al., 2006, 2012; Pothos and Busemeyer, 2009) for a synthesis of the "advantages of quantum uncertainty over the classical uncertainty". However, on a conceptual level, the notions of superposition and as well as entanglement are difficult to interpret in respect to human reasoning and choice formation. The problem of interpretation of these concepts is far from its final elucidation. Therefore, we prefer to justify the usage of quantum formalism by its mathematical simplicity. This argument might be surprising, because the quantum mechanics is always presented as one of the most complicated and even mystical scientific theories. However, this complexity lies merely in the 
foundations of quantum mechanics; its mathematical formalism (especially for the finite-dimensional state spaces used in the quantum information) is just about linear algebra, in particular, all dynamical equations are linear.

The state space of the proposed general model of decision making has a two level tensor product structure: the first level of the tensor product corresponds to the possible actions of a fixed agent and the second level unifies the state spaces of the agents, participating in the decision making task, thus providing an integrated model of agents' decision making. Along with the standards of quantum information, the tensor product state spaces contain special states which are qualified as entangled states. Entanglement encodes non-separability. ${ }^{17}$ Entanglement related to the first level tensor product encodes non-separability of actions of each concrete agent, say Alice, towards other agents, e.g. Bob, Natasha, John, etc. In the entangled mental state Alice cannot separate the choice of her action course, e.g., towards Bob, from the selection of her actions towards Natasha, John, ... . Entanglement related to the second level tensor product encodes non-separability of actions of agents towards each other. As was pointed out in Remark 2, the notion of entanglement is one of the most difficult interpretational issues of quantum mechanics. In this paper we proceed pragmatically, where entanglement is used to sustain a consistent mathematical modeling of non-separability of decisions, see Remark 2.

Finally, to obtain master equations describing evolution of the combined preference state of all the decision makers, we utilized an algebra of qubit operators of creation and annihilation, cf. Frydryszak (2011). Such a qubit algebra combines fermionic and bosonic commutation rules. The first type, anticommutation, represents the mutual exclusivity context for actions of the fixed agent towards another fixed agent and the second type, commutation, describes the coexistence of the preferences for actions towards different agents and agents towards each other. This algebra provides a possibility to formulate the state dynamics in the quantum-like manner, similarly to the standard equations used in quantum physics and based on algebras of fermionic and bosonic creation and annihilation operators. We remark that in standard quantum physics the qubit algebra did not attract so much interest. Nevertheless, it might happen that decision making and applications to cognitive psychology, sociology, economics, and finance will be the future areas of real applications of the qubit algebra of creation-annihilation operators. This paper could be treated a methodological introduction of the

\footnotetext{
${ }^{17}$ See Zorn and Smith $(2011)$ and Khrennikova $(2014,2015,2016)$ on a discussion about the representation of non-separability in political science with the aid of entanglement; see also Dzhafarov and Kujala $(2012,2014)$ for a connection of selective influences in psychophysics with the formalism of quantum entanglement.
} 
application of qubit algebra in human decision making processes in different contexts.

\section{Appendix: Bosons and fermions}

Quantum systems are divided into two classes, bosons (e.g., photons, quanta of the electromagnetic field) and fermions (e.g., electrons). Any number of bosons can occupy any fixed state and not more than one fermion can occupy any fixed state. This is the essence of the Pauli exclusion principle ${ }^{18}$. This principle is a postulate, and cannot be derived from the "natural physical principles". Theoretically, there is also a third class of possibilities. Let $m$ be a fixed natural number. Then it is said that a class of systems follows $m$-parastatistics, if not more than $m$ systems of this class can occupy a fixed state. Para-statistics were well studied in quantum foundations, but it is known that quantum systems do not follow any of para-statistics, different from the statistics of bosons or fermions. At the same time there are no reasons to assume that the same should hold in the applications to the problems of cognition, it might well be the case that some new para-statistics can arise. Moreover, various combinations of these statistics can naturally surface, as we have shown in this paper.

The states of bosons and fermions have to satisfy to different types of symmetries. This implies an existence of different commutation relations for the operators representing the processes of creation $a_{j}^{\star}$ and annihilation $a_{j}$ of bosons and fermions, respectively. For bosonic operators, we obtain:

$$
\left[a_{i}, a_{j}\right]=\left[a_{i}^{\star}, a_{j}^{\star}\right]=0,\left[a_{i}^{\star}, a_{j}\right]=\delta_{i j},
$$

where for any pair of operators $A, B,[A, B]=A B-B A$ is their commutator. For fermionic operators, we have:

$$
\left\{a_{i}, a_{j}\right\}=\left\{a_{i}^{\star}, a_{j}^{\star}\right\}=0,\left\{a_{i}^{\star}, a_{j}\right\}=\delta_{i j},
$$

where for any pair of operators $A, B,\{A, B\}=A B+B A$ is their anticommutator.

Remark: on the social meaning of the usage of fermionic and bosonic states

Bosonic states are displayed by agents in settings, where cooperation between them as well as inseparability of they decisions is possible. They also

\footnotetext{
${ }^{18} \mathrm{Cf}$. Ballentine (2014) for a general introduction.
} 
allow to encode the inseparability of decisions on the level of each single agent. In the setting of the proposed example (if we consider dichotomous choices in the form yes/no) in respect to the different decision-making tasks, the state vector of choices of two parties is denoted as $x=\left(x_{1}, x_{2}\right), x_{j}=0,1$. The bosonic properties of the decision operators of creation and annihilation imply that $a_{1}^{\star}, a_{1}$ commute with $a_{2}^{\star}, a_{2}$. As such, the compound state dynamics generated by these operators does not depend on the order of choice considerations by the political parties. When $P_{1}$ (one party) reflects towards cooperation, in the operational formalism this consideration is encoded in the application of the creation operator $a_{1}^{\star}$ to the state $\psi$. When $P_{2}$ (another party), for example, decides towards non-cooperation, in the operator formalism the annihilation operator $a_{2}$ is applied to the state $a_{1} \psi$, i.e., the output of these reflections is the state $\phi=a_{2} a_{1}^{\star} \psi$. The same output state would be generated if the parties' reflections take place in an opposite chronological order, i.e. $\phi=a_{1}^{\star} a_{2} \psi$. Hence, by applying the bosonic algebra for inter-party reflections, we construct a model, in which the order of reflections of the involved parties does not matter. One can say that the parties decide on their strategies independently. At the same time the parties' state $\psi$ can be also an entangled state, in this case any decision of, e.g., $P_{1}$ (represented in action of, e.g., $\left.a_{1}^{\star}\right)$ alters the state of the compound system.

Fermionic operators allow to model the decision making of agents in one qubit states. The properties of the fermionic operators allow to encode the $(0,1)$, i.e. dichotomicity of decision outcomes. For multiple outcome possibilities bosonic operators would be in use.

\section{Acknowledgements}

I would like to thank A. Khrennikov for discussions and consultations on the mathematical formalism of quantum theory, in particular, for kindly pointing out that the mixed fermionic-bosonic algebra of creation and annihilation operators is known in quantum information theory as the qubit algebra of creation and annihilation operators and suggesting the relevant references to this field (Frydryszak, 2011). I also would like to thank the anonymous reviewers for their kind remarks on the usage of fermionic-bosonic algebra of creation and annihilation operators, as well as their remarks on the generalizability of the proposed model to broader contexts of decision making.

\section{References}

Accardi, L, Khrennikov, A, Ohya, M. (2008). The problem of quantumlike representation in economy, cognitive science, and genetics. Quantum 
Bio-Informatics II, From Quantum Information to Bio-Informatics,1-8.

Accardi, L, Khrennikov, A, Ohya, M. (2009). Quantum Markov model for data from Shafir-Tversky experiments in cognitive psychology. Open Systems and Information Dynamics, 16, 371-385.

Aerts, D., Sozzo, S., Tapia, J. (2012). A Quantum Model for the Ellsberg and Machina Paradoxes. Quantum Interaction. Lecture Notes in Computer Science, 7620, pp 48-59.

Asano M, Ohya M, Khrennikov A. (2011a). Quantum-Like Model for Decision Making Process in Two Players Game - A Non-Kolmogorovian Model. Foundations of Physics, 41: 538-548.

Asano M., Ohya, M., Tanaka,Y., Basieva, I., Khrennikov, A. (2011b) Quantum-like model of brain's functioning: decision making from decoherence, Journal of Theoretical Biology, 281, 56-64.

Asano, M., Ohya, M., Tanaka, Y., Basieva,I., Khrennikov, A. (2011c) On application of Gorini-Kossakowski-Sudarshan-Lindblad equation in cognitive psychology. Open Systems and Information Dynamics, 18, 55-69.

Asano, M., Ohya, M., Tanaka, Y., Basieva, I., Khrennikov, A. (2012) Quantum-like dynamics of decision-making, Physica A, 391, 2083-2099.

Asano, M., Khrennikov, A., Ohya, M., Tanaka, Y., Yamato, I. (2014). Violation of contextual generalization of the Leggett-Garg inequality for recognition of ambiguous figures. Physica Scripta T163, 014006.

Asano M., Basieva, I., Khrennikov, A., Ohya, M., Tanaka,Y. (2016) A Quantum-like model of selection behavior. Journal of Mathematical Psychology,(forthcoming).

Bagarello, F. (2012) Quantum dynamics for classical systems: with applications of the Number operator, Wiley Ed., New York.

Bagarello, F. (2015) An operator view on alliances in politics, SIAM Journal of Applied Mathematics, 75(2), 564-584.

Bagarello, F., Haven, E. (2016). First results on applying a non-linear effect formalism to alliances between political parties and buy and sell dynamics. Physica A, 444 (403-414)

Ballentine, L. (2014) Quantum Mechanics: A Modern Development. (2nd ed.) World Scientific Publishing.

Basieva I., Khrennikov, A., Ohya, M., Yamato, I. (2011) Quantum-like interference effect in gene expression glucose-lactose destructive interference. Systems and Synthetic Biology, 5(1):59-68.

Birnbaum, M. H. (2008). New paradoxes of risky decision making. Psychological Review, 115(2), 463-501.

Boyer-Kassem T, Duchene S, Guerci E. (2016a), Testing quantum-like models of judgment for question order effect, Mathematical Social Sciences, 80: $33-46$. 
Boyer-Kassem T, Duchene S, Guerci E. (2016b ), Quantum-like models cannot account for the conjunction fallacy, Theory and Decision, (forthcoming).

Bravyi, S. and Kitaev, A. (2002) Fermionic quantum computation. Annals of Physics, 298, 210-226.

Busemeyer, J. B., Wang, Z., Townsend, J. T. (2006) Quantum dynamics of human decision making, Journal of Mathematical Psychology, 50, 220-241.

Busemeyer, J.R., Bruza, P. D. (2012) Quantum models of cognition and decision, Cambridge University Press, Cambridge.

Conte, E., Todarello, O., Federici, A., Vitiello, F., Lopane, M., Khrennikov, A., and Zbilut, J. P. (2007) Some remarks on an experiment suggesting quantum-like behavior of cognitive entities and formulation of an abstract quantum mechanical formalism to describe cognitive entity and its dynamics. Chaos, Solitons and Fractals, 31(5), 1076-1088.

Conte, E., Khrennikov, A., Todarello, O., Federici, (2008) A preliminary experimental verification on the possibility of Bell Inequality violation in mental states. NeuroQuantology 6 (3), 214-221.

Conte, E., Khrennikov, A., Todarello, O., Federici, A., Mendolicchio, Zbilut, J. P. (2009) Mental state follow quantum mechanics during perception and cognition of ambiguous figures. Open Systems and Information Dynamics, $16,1-17$.

Dzhafarov, E.N., Kujala, J.V. (2012) Quantum entanglement and the issue of selective influences in psychology: An overview. In Quantum Interaction 6th International Symposium, QI 2012, Paris, France, June 27-29, 2012; (Busemeyer at al., eds.) Lecture Notes in Computer Science, 7620, 184-195.

Dzhafarov, E.N., Kujala, J.V. (2014). On selective influences, marginal selectivity, and Bell/CHSH inequalities. Topics in Cognitive Science 6, 121128.

Ellsberg, D. (1961) Risk, Ambiguity, and the Savage Axioms. Quarterly Journal of Economics 75 (4): 643-669.

Erev, I., and Ert, E. (2016) From anomalies to forecasts: Toward a descriptive model of decisions under risk, under ambiguity, and from experience. Preprint.

Feynman, R. and Hibbs, A. (1965). Quantum Mechanics and Path Integrals (McGraw-Hill, New York).

Frydryszak, A. (2011) Pure state entanglement in terms of nilpotent variables: $\eta$-toolbox. In: Quantum Dynamics and Information. Olkiewicz, R., Cegla, W., and Frydryszak, A., Garbaczewski, P., Jakobzyk, L. (eds), WSP, Singapore, pp. 79-112.

Hameroff, S., Penrose, R. (2014) Consciousness in the Universe: A review of the Orch OR' theory. Physics of Life Reviews, 11(1), 39-78. 
Haven E, Khrennikov A. (2009). Quantum mechanics and violation of the sure-thing principle: the use of probability interference and other concepts. Journal of Mathematical Psychology 53, 378-388.

Haven, E. and Khrennikov, A. (2016) Statistical and subjective interpretations of probability in quantum-like models of cognition and decision making. J. Math. Psych. http://www.sciencedirect.com/science/article/pii/S0022249616000213

Kahneman D., Tversky A., eds. (2000), Choices, Values and Frames, New York : Cambridge University Press and the Russell Sage Foundation.

Khrennikova, P. (2014a) A quantum framework for 'Sour Grapes' in cognitive dissonance. In Quantum Interaction 7th International Conference, QI 2013, Leicester, UK, July 25-27, 2013; Lecture Notes in Computer Science (Atmanspacher H., et. al., eds.),8369, 270-280 .

Khrennikova P. (2014b) Order effect in a study on voters' preferences: quantum framework representation of the observables, Physica Scripta, 0104010, $1-8$.

Khrennikova, P., Haven, E., Khrennikov, A. (2014) An application of the theory of open quantum systems to model the dynamics of party governance in the US Political System, International Journal of Theoretical Physics, 53(4), 1346-1360.

Khrennikova, P. (2015) Quantum like Modelling of the Nonseparability of Voters' Preferences in the U.S. Political System. In Quantum Interactions, 8th International Conference, QI 2014, Filzbach, Switzerland, June 30 -July 3, 2014; (Atmanspacher et al., eds.) Lecture Notes in Computer Science, 8951, 196-209.

Khrennikova, P., Haven, E. (2016) Instability of political preferences and the role of mass-media: a dynamical representation in a quantum framework. Philosophical Transactions of the Royal Society A, 374, 20150106.

Khrennikova, P. (2016) Quantum dynamical modeling of competition and cooperation between political parties: the coalition and non-coalition equilibrium model. J. Math. Psych., to be published.

Loubenets, E. R. (2012) Local quasi hidden variable modelling and violations of Bell-type inequalities by a multipartite quantum state. Journal of Mathematical Physics, 53, 022201.

Loubenets, E. R. (2015) Context-invariant quasi hidden variable (qHV) modelling of all joint von Neumann measurements for an arbitrary Hilbert space. Journal of Mathematical Physics, 56, 032201.

Machina, M.J. (2009). Risk, Ambiguity, and the Dark-dependence Axioms. Am. Econ. Rev. 99(1), 385-392.

Ohya M, Volovich I. (2011). Mathematical foundations of quantum information and computation and its applications to nano- and bio-systems. Heidelberg-Berlin-New York: Springer. 
Plotnitsky, A. (2009). Epistemology and probability: Bohr, Heisenberg, Schrödinger and the nature of quantum-theoretical thinking. HeidelbergBerlin-New York: Springer.

Plotnitsky, A. (2014). Are quantum-mechanical-like models possible, or necessary, outside quantum physics? Phys. Scr., T163, 014011.

Pothos, E., Busemeyer,J. R. (2009) A quantum probability explanation for violations of 'rational' decision theory. Proceedings of Royal Society B, 276, 2171-2178.

Savage, L. J. (1954) The foundations of statistics. New York, Wiley.

Sozzo, S. (2014). A Quantum probability explanation in Fock space for borderline contradictions. Journal of Mathematical Psychology, 58(1), 1-12.

Tversky, A. and Kahneman, D. (1974), Judgement under Uncertainty: Heuristics and Biases, Science, 185 (4157), 1124-1131.

Tversky, A., Kahneman D. (1981), The framing of decisions and the psychology of choice, Science, 211, 453-458.

Tversky A., Kahenman D. (1983) Extensional Versus Intuitive Reasoning: The Conjunction Fallacy in Probability Judgement. Psychological Review, 90(4), pp. 293-315.

Tversky A., Shafir E. (1992) The disjunction effect in choice under uncertainty, Psychological science, 3(5), 305-309.

von Neumann, J., Morgenstern, O. (1953) Theory of Games and Economic Behavior. Princeton, NJ. Princeton University Press.

Zorn, C., Smith, C. (2011) Pseudo-Classical Nonseparability and Mass Politics in Two-Party Systems. In Quantum Interactions, 5th International Symposium, QI 2011, Aberdeen, UK, June 26-29, 2011;(Song D et. al., eds.). Lecture Notes In Computer Science, 7052, 83-94.

Wang, Z. and Busemeyer, J. R. (2013) A quantum question order model supported by empirical tests of an a priori and precise prediction. Topics in Cognitive Science, 5, 689-710.

White, L. C., Pothos, E.M., Busemeyer, J. R. (2013) A quantum probability perspective on the nature of psychological uncertainty. M. Knauff, M. Pauen, N. Sebanz, I. Wachsmuth (Eds.), Proceedings of the 35th annual conference of the cognitive science society, Cognitive Science Society, Austin TX, pp. 15991604.

White., L. C., Pothos, E.M., Busemeyer, J. R. (2014) Sometimes it does hurt to ask: The constructive role of articulating impressions, Cognition, 133 (1), 4864.

White.,L. C., Pothos, E.M., Busemeyer, J. R. (2015) Insights from quantum cognitive models for organizational decision making, Journal of Applied Research in Memory and Cognition, Modeling and Aiding Intuition in Organizational Decision Making, 4, N 3, 229238 